\title{
A 2D Mesh-free Simulation of the Particle Adhesion in a Plastic Cyclone
}

DOI:

$10.1177 / 0954406218758794$

\section{Document Version}

Accepted author manuscript

Link to publication record in Manchester Research Explorer

\section{Citation for published version (APA):}

Zhou, Y., See, T., Zhong, S., Liu, Z., \& Li, L. (2019). A 2D Mesh-free Simulation of the Particle Adhesion in a Plastic Cyclone. Institution of Mechanical Engineers. Proceedings. Part C: Journal of Mechanical Engineering Science, 233(2), 649-663. https://doi.org/10.1177/0954406218758794

\section{Published in:}

Institution of Mechanical Engineers. Proceedings. Part C: Journal of Mechanical Engineering Science

\section{Citing this paper}

Please note that where the full-text provided on Manchester Research Explorer is the Author Accepted Manuscript or Proof version this may differ from the final Published version. If citing, it is advised that you check and use the publisher's definitive version.

\section{General rights}

Copyright and moral rights for the publications made accessible in the Research Explorer are retained by the authors and/or other copyright owners and it is a condition of accessing publications that users recognise and abide by the legal requirements associated with these rights.

\section{Takedown policy}

If you believe that this document breaches copyright please refer to the University of Manchester's Takedown Procedures [http://man.ac.uk/04Y6Bo] or contact uml.scholarlycommunications@manchester.ac.uk providing relevant details, so we can investigate your claim.

\section{OPEN ACCESS}




\title{
A 2D Mesh-free Simulation of the Particle Adhesion in a Plastic
}

\section{Cyclone}

\author{
Yuanye Zhou ${ }^{1,2}$, Tianlong See ${ }^{2,3}$, Shan Zhong ${ }^{1,2}$, Zhu Liu' ${ }^{2,3}$, Lin $\mathrm{Li}^{1,2}$ \\ ${ }^{1}$ School of Mechanical, Aerospace, and Civil Engineering, The University of Manchester, \\ Oxford Road, Manchester, M13 9PL, United Kingdom \\ ${ }^{2}$ Laser Processing Research Centre, The University of Manchester, Oxford Road, Manchester, \\ M13 9PL, United Kingdom \\ ${ }^{3}$ School of Materials, The University of Manchester, Oxford Road, Manchester, M13 9PL, \\ United Kingdom \\ *Corresponding author at: School of Mechanical, Aerospace and Civil Engineering, The \\ University of Manchester, Oxford Road, M13 9PL, United Kingdom \\ E-mail address: yuanye.zhou@manchester.ac.uk
}

\begin{abstract}
Although the particle adhesion is a classic problem in cyclones, it is not clearly understood in previous studies. This study was set out to determine factors affecting the micro-particle adhesion in an Acrylonitrile Butadiene Styrene (ABS) cyclone by developing a mesh-free simulation method to predict the dynamic motion of a single particle in a 2D horizontal section of the cyclone with the presence of the wall boundary layer. Simulation results showed that the centrifugal force played a minor role on the particle adhesion but it was important on the transportation of the particle. In contrast, the electrostatic force was important on the particle adhesion but it was not important on the transportation of the particle. Moreover, simulation results suggested that the particle adhesion can be reduced by increasing the inlet velocity of the cyclone or by increasing the coefficient of restitution $(\mathrm{CoR})$ of the particle-wall collision. In contrast, the particle adhesion can be increased by increasing the radius of the cyclone, the particle charge and the coefficient of friction $(\mathrm{CoF})$. Furthermore, simulation results on the effect of the inlet velocity and particle charge were validated by experimental results.
\end{abstract}


Key words: Particle, Adhesion, Cyclone, Simulation, Mesh-free

\section{Nomenclature}

$a$

$b$

$d$

$D$

De

$H c$

$L$

$R a$

$S$

th

$U_{\theta}$

$U_{\text {in }}$

$U_{r}$

$x$

$\theta$

$\dot{\theta}$

$r$

$r_{0}$

$\dot{r}$

$F S_{\theta}$

$F S_{r}$

FC

FE
Width of cyclone inlet [m]

Height of cyclone inlet [m]

Diameter of cyclone tip [m]

Diameter of cyclone cylindrical body [m]

Diameter of cyclone vortex finder [m]

Length of cyclone cylindrical body [m]

Length of cyclone conical body [m]

Surface roughness [m]

Length of cyclone vortex finder [m]

Thickness of vortex finder [m]

Flow tangential velocity $\left[\mathrm{m} \cdot \mathrm{s}^{-1}\right]$

Inlet velocity of cyclone separator $\left[\mathrm{m} \cdot \mathrm{s}^{-1}\right]$

Flow radial velocity $\left[\mathrm{m} \cdot \mathrm{s}^{-1}\right]$

Diameter of particle [m]

Tangential location of particle [1]

Tangential velocity of particle $\left[\mathrm{s}^{-1}\right]$

Radial location of particle [m]

Initial radial location of particle [m]

Radial velocity of particle $\left[\mathrm{m} \cdot \mathrm{s}^{-1}\right]$

Tangential aerodynamic force $\left[\mathrm{m} \cdot \mathrm{s}^{-2}\right]$

Radial aerodynamic force $\left[\mathrm{m} \cdot \mathrm{s}^{-2}\right]$

Centrifugal force $\left[\mathrm{m} \cdot \mathrm{s}^{-2}\right]$

Electrostatic force $\left[\mathrm{m} \cdot \mathrm{s}^{-2}\right]$ 


$\begin{array}{ll}F V & \text { van der Waals force }\left[\mathrm{m} \cdot \mathrm{s}^{-2}\right] \\ F C & \text { Capillary force }\left[\mathrm{m} \cdot \mathrm{s}^{-2}\right] \\ F F & \text { Friction force }\left[\mathrm{m} \cdot \mathrm{s}^{-2}\right] \\ d t & \text { Time step }[\mathrm{s}] \\ \tau & \text { Particle relaxation time }[\mathrm{s}] \\ \rho_{p} & \text { Particle density }\left[\mathrm{kg} \cdot \mathrm{m}^{-3}\right] \\ \mu & \text { Flow dynamic viscosity }\left[\mathrm{kg} \cdot \mathrm{m}^{-1} \bullet \mathrm{s}^{-1}\right] \\ \varepsilon_{0} & \text { Dielectric constant of free } \mathrm{space}\left[\mathrm{kg}^{-1} \cdot \mathrm{m}^{-3} \cdot \mathrm{A}^{2} \cdot \mathrm{s}^{4}\right] \\ q & \text { Particle electrostatic charge }[\mathrm{A} \cdot \mathrm{s}] \\ e & \text { Elementary charge }[\mathrm{A} \cdot \mathrm{s}] \\ R & \text { Radius of cyclone separator cross section }[\mathrm{m}] \\ Q & \text { Cumulative particle charge }[\mathrm{A} \cdot \mathrm{s}] \\ \dot{N} & \text { Number flux rate of particles }\left[\mathrm{s}^{-1}\right] \\ \dot{m} & \text { Mass flux rate of particles }\left[\mathrm{kg} \bullet \mathrm{s}^{-1}\right]\end{array}$
Abbreviations
$\mathrm{CoF}$
Coefficient of Friction
CoR
Coefficient of Restitution
TCG
Triboelectric Charge Generator

\section{Introduction}

In the particle handling area, cyclones are popular used devices, due to its simple geometry and robust performance. During operation, particle adhesion can block the cyclone, which is unwanted. Therefore, it is necessary to avoid particle adhesion in cyclones (O'Callaghan and Cunningham, 2005). So far, in the industry application, some methods to reduce particle adhesion are used. For example, the use of jet flow 
tube to blow away particle adhesion (Huang et al., 2013; He et al., 2014); adding a portable rod to scrap and remove particle adhesion (Mozley, 1979); replacing the cone tip with a vibrating rubber nozzle in a commercial vacuum cleaner.

A number of academic investigations have been carried out on analysing the particle adhesion in cyclones. Some of them dealt with visualizing particle adhesion patterns and identifying locations of particle adhesion patterns (Yuu et al., 1978; Bogodage and Leung, 2016). Houben (2011) discussed various forces that affect the particle adhesion in the cyclone. His findings suggested that the dominating forces on particle adhesion in a cyclone were the capillary force, the van der Waals force, the electrostatic force and the aerodynamic force, which agreed well with other researchers' findings of the particle adhesion on the flat surface (Jones et al., 2002; Bowling, 1985; Corn, 1961; Cleaver and Yates, 1973; Mittal and Jaiswal, 2015). However, the flow condition in the cyclone was more complicated than the flat surface. For example, a sudden increase in particle adhesion behind the vortex end was reported by Peng et al. (2007). Zhou et al. (2017) suggested that the sudden increase in particle adhesion was due to low wall shear stress, i.e. weak aerodynamic force, behind the vortex end at the cyclone tip. In addition, Song et al. (2017) found that the amount of particle adhesion in the cyclone was not uniform on the outer surface of vortex finder, which was caused by the local uneven particle concentration.

In the cyclone, the particle adhesion is a dynamic process that involved the transportation of particles from a faraway location to the wall surface. In previous study (Houben, 2011), particle adhesion is mainly considered in a steady state, that particle is on the surface. In the steady state, van der Waals force is the dominate force on particle adhesion. However, from the view of dynamic process, the kinetic energy of particle is also important. If particle keeps moving, adhesion will not form. Whether electrostatic force and centrifugal force can increase the kinetic energy of particle is unclear. Therefore, the electrostatic force and the centrifugal force might be more important, because the electrostatic force was found to account for the transportation of particles to the wall (Lim et al., 2012) and the centrifugal force 
pushed particles moving towards the wall.

In order to explore the transportation of particles from a faraway location to the wall surface in the cyclone, the computational fluid dynamics (CFD) simulation was a popular choice (Wang et al., 2006; Chu et al., 2011). Although results of the CFD simulation were able to provide adequate knowledge on the particle trajectory and two-way coupled interactions, it was limited to predict the particle adhesion, because the particle adhesion was usually formed by micro-particles, of which the sizes were much smaller than mesh sizes on the wall in the CFD simulation. It meant that the CFD simulation usually overestimates the flow velocity in the wall boundary layer for the particle adhesion, unless using enough small mesh sizes or introducing a damp factor to correct the flow velocity (Houben et al., 2012).

Even though CFD simulation can be improved for particle transportation and adhesion in the cyclone, effects of the electrostatic force and centrifugal force on particle adhesion have not been discussed in detail. Therefore, there is need to understand effects of the electrostatic force and centrifugal force on the particle adhesion in the cyclone.

In this study, to overcome the limitation of the mesh size in the CFD simulation on the particle adhesion in the cyclone, a mesh-free simulation method was proposed. The flow velocity was continually interpolated by combining our previous CFD results with a linear flow velocity profile in the wall boundary layer. According to the interpolated flow velocity, the dynamic process of the particle adhesion that involved the transportation of a single particle from a faraway location to the wall surface in a 2D horizontal section of the cyclone was simulated. The effect of the electrostatic force and centrifugal force on the particle adhesion was discussed based on the particle trajectory. Besides, factors affecting the particle adhesion in the cyclone were investigated. Experimental validations of the simulation were also conducted.

\section{Material and methods}




\subsection{Particle and cyclone}

As plaster particles can easily form the adhesion, Thistle Dura-Finish plaster, manufactured by British Gypsum Ltd. was used in the experiment. The density of the plaster particle is about $2300 \mathrm{~kg} / \mathrm{m}^{3}$. Before the experiment, plaster particles were stored in a sealed tank at the room temperature, so that particles were not wetted by the humidity. Samples of particles were diluted by air in a sedimentation tank and the particle size (in terms of dynamically equivalent diameter) was measured by a particle sizer (TSI 3321). The average size of plaster particles was $1.13 \mu \mathrm{m}$.

The cyclone used in this study was made by Acrylonitrile Butadiene Styrene (ABS). Its configuration consisted of a tangential inlet, a cone shape body and a vortex finder, as shown in Figure 1. The dimensions of the cyclone are listed in Table 1. In this study, the particle adhesion in the cyclone was focused on the conical part of the cyclone, because most of particle adhesion occurred on this part. The conical part had a surface roughness of $R a=7.63 \mu$ m (measured by Keyence VK-X200K 3D Laser Scanning Microscope). Before the experiment, the cyclone was washed by the water and dried in the air to have a clean surface.

Table 1 Dimensions of the cyclone used in the experiment

\begin{tabular}{cccc}
\hline Feature & Dimension, $\mathrm{mm}$ & Feature & Dimension, $\mathrm{mm}$ \\
\hline $\mathrm{a}$ & 5 & $\mathrm{~S}$ & 13.55 \\
$\mathrm{~b}$ & 11.6 & $\mathrm{Hc}$ & 12.1 \\
$\mathrm{D}$ & 35 & $\mathrm{~L}$ & 87.11 \\
$\mathrm{De}$ & 8.54 & $\mathrm{~d}$ & 6.6 \\
th & 1 & & \\
\hline \multicolumn{4}{c}{ * Measurement errors are within $0.01 \mathrm{~mm}$}
\end{tabular}




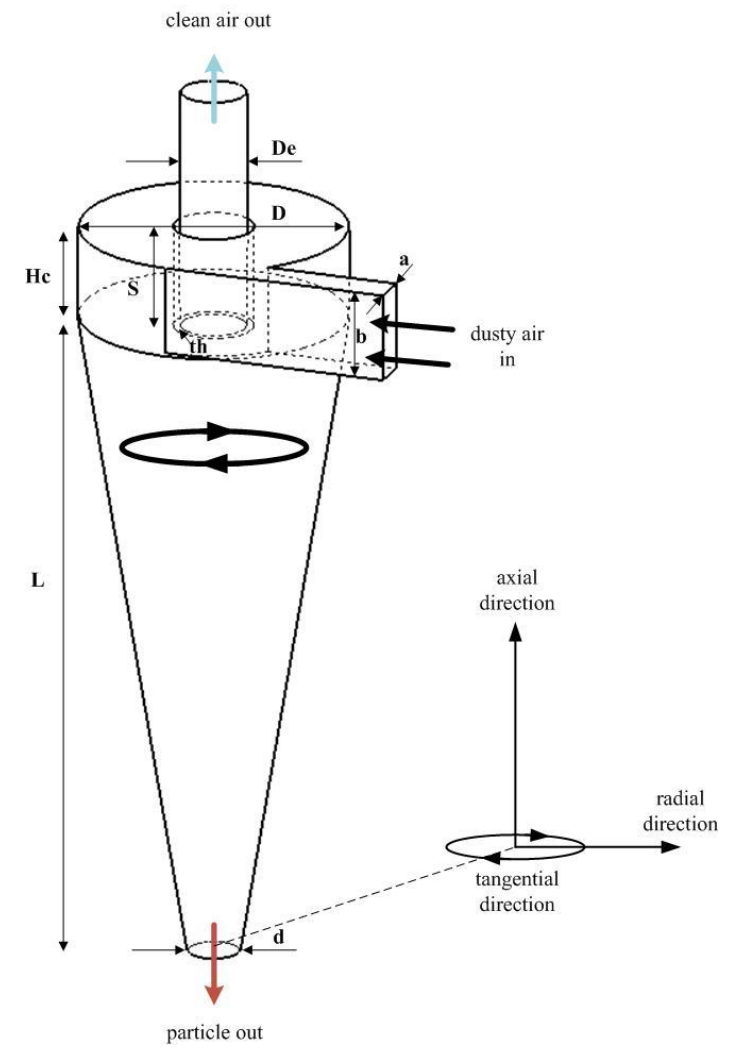

Figure 1 Illustration of a typical cyclone and its key dimensions

\subsection{Experimental methods}

The experiment test was designed to validate the simulation. The test rig consisted of a triboelectric charge generator (TCG), a cyclone, a fibre filter, an air pump with a pump voltage adjustor and sensors for pressure and mass flow rate measurement, as shown in Figure 2.

In order to generate the particle charge, the TCG was made from a commercial vacuum cleaner. During the experiment, the TCG was operating at a fixed air flow rate $(2000 \mathrm{~L} / \mathrm{min})$. Particles were prepared and fed into the TCG through the shaker (model V20/PA100 manufactured by Gearing \&Watson Ltd.) at a stable load rate. On the side wall of the TCG, a hole (around $10 \mathrm{~mm}$ diameter) was drilled. A tube with a diameter of $10 \mathrm{~mm}$ was placed as a bridge to connect the cyclone to the TCG. By running the air pump (model Secoh JDK-S-500, ATAC Ltd., Maidstone, UK), a small portion of the dusty air $(105-150 \mathrm{~L} / \mathrm{min})$ would move into the cyclone from the 
TCG.

During the experiment, the mass flow rate, the pressure drop and the particle charge were measured. The mass flow sensor (model TSI 40241, TSI Ltd.) was located behind a fibre filter. The pressure sensor (Sensortechnics HDIM series, Sensortechnics Ltd., Rugby, UK) was placed across the cyclone separator for pressure drop measurement. Both the mass flow sensor and the pressure sensors produced voltage signals. An NI card (model NI PCI-6221, National Instruments Ltd., Newbury, UK) was used to record the voltage signals, with a sampling rate of $200 \mathrm{~Hz}$. To quantify the particle charge, a short section of the inlet tube of the cyclone separator was made by conductive steel, as shown in Figure 3. It allowed the particle charge to be measured by the electrostatic charge sensor, which was a digital coulomb meter (model XJC-205-010F, full scale \pm 1999 nC, Fisher-Scientific, UK). It was noted that the digital coulomb meter read the cumulative particle charge over time. The duration of each test was 20 minutes.

After the experiment, the level of particle adhesion was indicated by its weight, which was measured by a digital weight scale (model Kern PCB 100-3, RS-online, UK) with a resolution of $0.001 \mathrm{~g}$. The environmental temperature and humidity during the experiment was $20 \pm 2{ }^{\circ} \mathrm{C}$ and $50 \pm 5 \% \mathrm{RH}$, respectively. The variation of room pressure was less than $5 \%$ of $101 \mathrm{kPa}$ over one year, according to the data from centre of atmospheric science in the university. Thus, the test environment condition is regarded as constant.

Effects of two factors were examined with this test rig. They were the particle charge and the inlet velocity of the cyclone. With the TCG, particles were charged due to triboelectric effect. The condition that particles had the charge was defined as the charged condition. In contrast, the TCG was removed so that the uncharged condition with zero particle charge was achieved. The variation of the inlet velocity of the cyclone was achieved by using the pump voltage adjustor to control the flow rate.

The inlet velocity and particle load rate for different tests were listed in Table 2. 
Table 2 Flow rate and particle load rate for different conditions

\begin{tabular}{ccc}
\hline & Inlet velocity, $\mathrm{m} / \mathrm{s}$ & Particle load rate, $\mathrm{g} / \mathrm{min}$ \\
\hline Effect of particle charge & 30 & $0.029-0.101$ \\
Effect of inlet velocity & $30-43$ & 0.101 \\
\hline
\end{tabular}

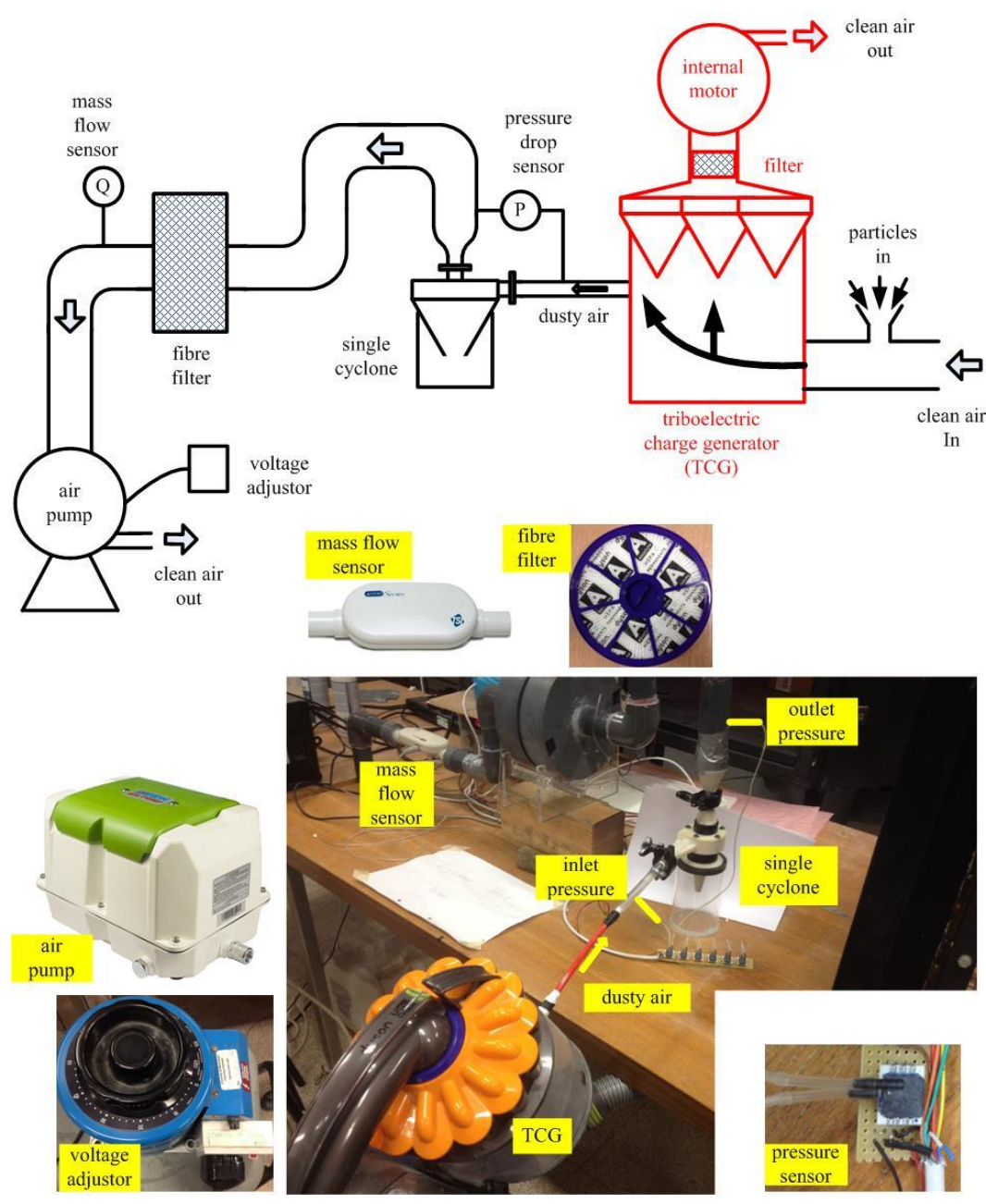

Figure 2 Experimental test rig
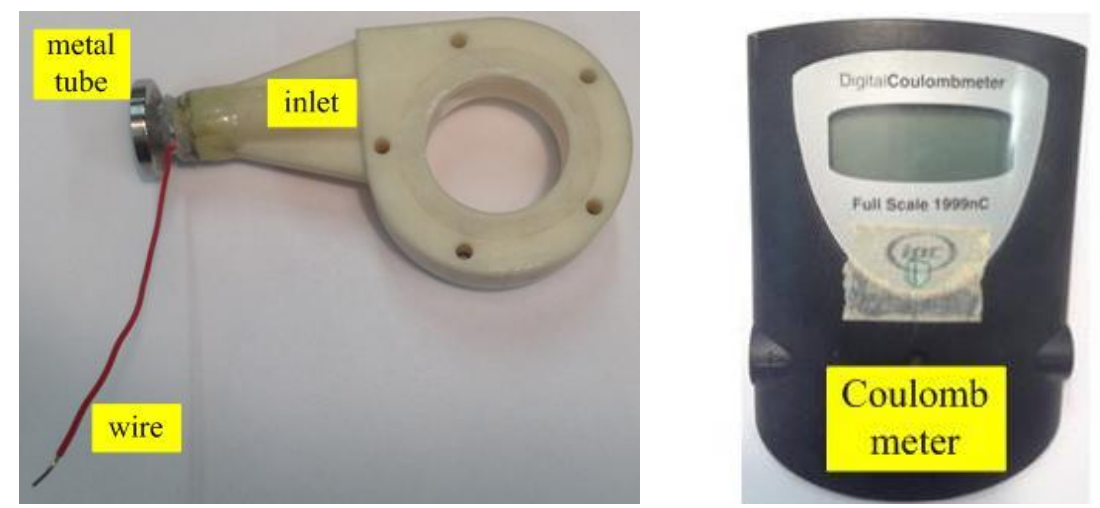

Figure 3 Measurement technique of particle charge 


\subsection{Simulation methods}

\subsubsection{D model of the particle dynamic motion in the cyclone}

As the particle dynamic motion in a cyclone was complicated, a simplified model, focusing on a single spherical particle moving in a horizontal cross section of a smooth cyclone, was proposed to describe the $2 \mathrm{D}$ particle dynamic motion in the cyclone, as shown in Figure 4. A fixed polar coordinate was defined to locate the particle. The $\theta$-direction was the tangential direction and $r$-direction was the radial direction. The flow was assumed to be swirling in the whole cross section.

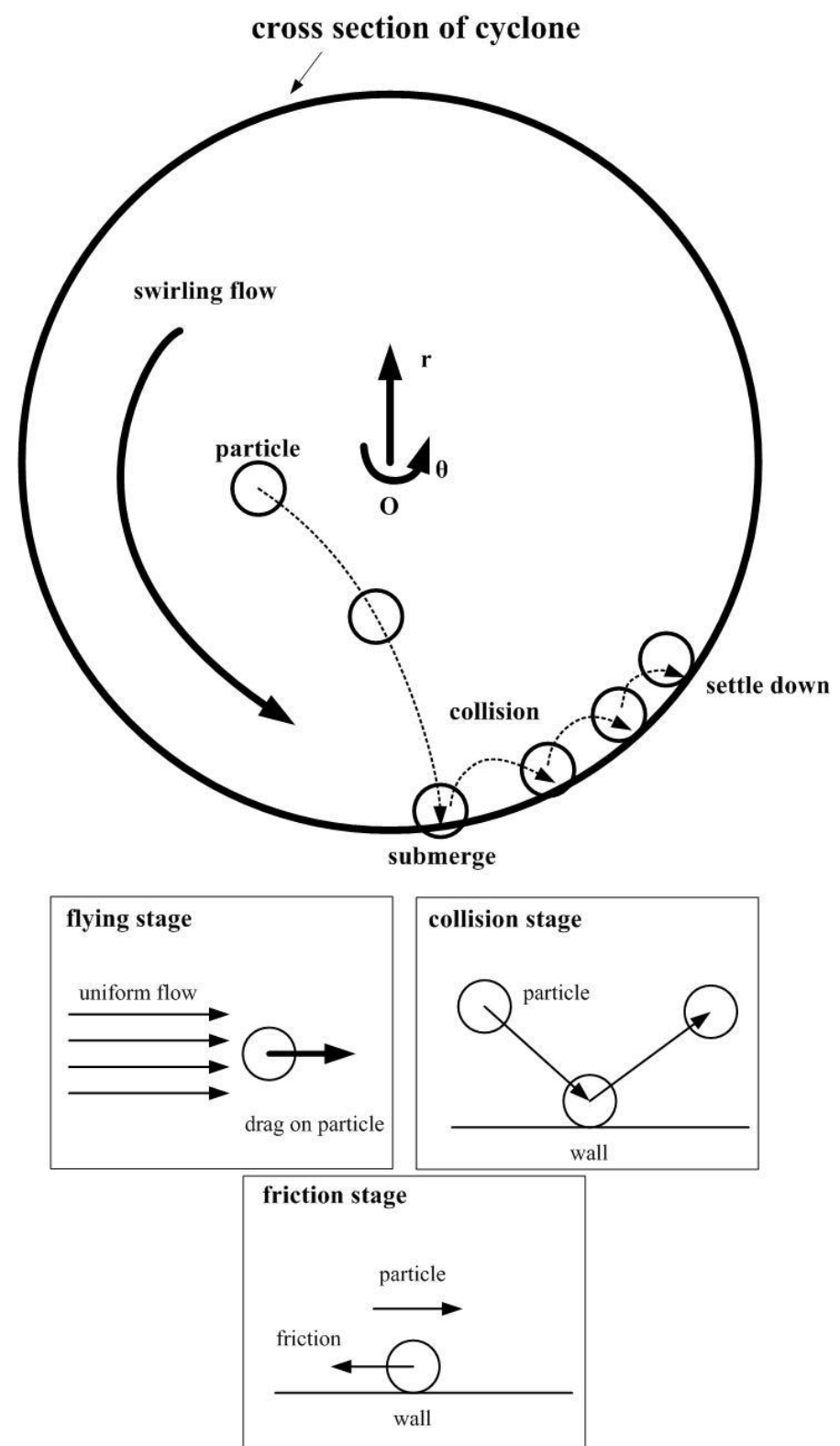


Figure 4 2D model of the particle dynamic motion in the cross section of the cyclone According to the model, the motion of the particle was classified into three stages. They were the flying stage, collision stage and friction stage. The flying stage was defined as the period when the particle did not contact with the wall. In this period, the particle was mainly affected by the air flow. The collision stage was the instantaneous period when the particle-wall collision occurred. The friction stage was the period when the particle was on the wall surface. The particle was allowed to be submerged into the wall surface at the collision stage and friction stage, as it can represent the deformation of the particle on the wall surface. The submerge distance was set to be $1 / 10$ of particle diameter.

The state of the particle was written by $\left[\begin{array}{llll}\theta & \dot{\theta} & r & \dot{r}\end{array}\right]$, where $\theta, r$ was the location of the particle centroid, and $\dot{\theta}, \dot{r}$ was the particle centroid velocity in the relevant direction. The forces acting on the particle in the cyclone were shown schematically in Figure 5. These forces included the aerodynamic forces in two directions $\left(F S_{\theta}, F S_{r}\right)$, the centrifugal force $(F C)$, the electrostatic force $(F E)$, the van der Waals force $(F V)$, the capillary force $(F C)$ and the friction $(F F)$. The gravity was neglected, as it much smaller than the aerodynamic forces in cyclone (Hoffmann and Stein, 2002). It was noted that all these forces were normalized to the particle mass.

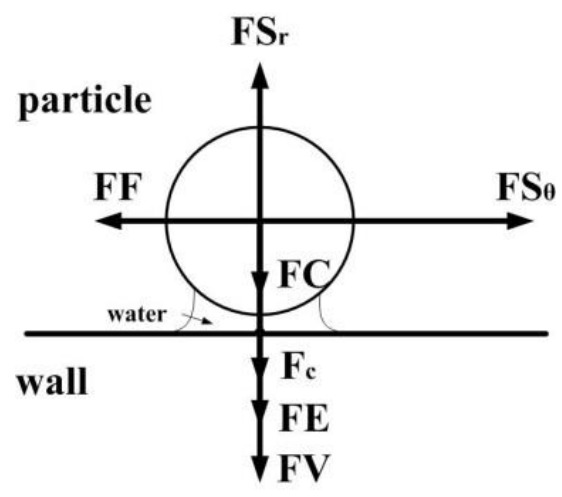

Figure 5 Forces acting on an invididual particle in the cyclone

In flying stage, Newton's second law is applied. In collision stage, elastic collision model is applied. In friction stage, a sliding detachment model (Wang, 1990) was 
adopted. The general equations of the particle dynamic motion were given as below:

$$
\left.\left[\begin{array}{c}
\theta \\
\dot{\theta} \\
r \\
\dot{r}
\end{array}\right]_{t+d t}=\left[\begin{array}{cccc}
1 & d t & 0 & 0 \\
0 & 1+\frac{F S_{\theta}}{r \dot{\theta}} d t & 0 & 0 \\
0 & 0 & 1 & d t \\
0 & 0 & 0 & 1+\frac{F S_{r}+F C+F E}{\dot{r}} d t
\end{array}\right]_{t}\right]_{t}
$$

$$
\left[\begin{array}{c}
\theta \\
\dot{\theta} \\
r \\
\dot{r}
\end{array}\right]_{t+d t}=\left[\begin{array}{cccc}
1 & 0 & 0 & 0 \\
0 & C o R & 0 & 0 \\
0 & 0 & 1 & 0 \\
0 & 0 & 0 & -\operatorname{CoR}
\end{array}\right]_{t}\left[\begin{array}{c}
\theta \\
\dot{\theta} \\
r \\
\dot{r}
\end{array}\right]_{t} \text { (Collision) }
$$

$$
\left.\left[\begin{array}{c}
\theta \\
\dot{\theta} \\
r \\
\dot{r}
\end{array}\right]_{t+d t}=\left[\begin{array}{cccc}
1 & d t & 0 & 0 \\
0 & 1+\frac{F S_{\theta}-F F}{r \dot{\theta}} d t & 0 & 0 \\
0 & 0 & 1 & d t \\
0 & 0 & 0 & 1+\frac{F S_{r}+F C+F E+F V+F c}{\dot{r}} d t
\end{array}\right]_{t}\right]_{t}
$$

Where $t$ is the time, $d t$ is the time step, and the CoR is the coefficient of restitution.

In this study, some simplifications on estimating forces were applied. The aerodynamic force was assumed to be the Stokes' drag, as the lift force was smaller than the Stokes' drag in an order of magnitude (Hoffmann and Stein, 2002). The near wall effect on the Stokes' drag was not considered, at it did not change the Stokes' drag in an order of magnitude (O’Neill, 1968). The electrostatic force was determined by the Coulomb's law between a single particle and a conducting infinitely long flat surface, as the particle was much smaller than the size of the wall curvature. The van der Waals force and capillary force were assumed to be zero, as they were constant in the experiment. Because, velocity is the integration of acceleration (force/mass) and position is the integration of velocity, a constant van der Waals force and capillary 
force will lead to a constant shift in velocity and position but remain the curve trend the same, compared with the zero assumption. Thus, the effect of van der Waals force and capillar force was regarded as the base value that was set to be zero. Actually, a lack of experimental data on determining the van der Waals force and capillary force limits an accurate prediction of these two forces.

According to above simplifications, forms of thoses forces (normalized to the particle mass) acting on the particle were written as below:

$$
\begin{aligned}
& F S_{\theta}=\frac{U_{\theta}-r \dot{\theta}}{\tau} \\
& F S_{r}=\frac{U_{r}-\dot{r}}{\tau} \\
& F C=r \dot{\theta}^{2} \\
& F E=\frac{3 q^{2}}{8 \pi^{2} \varepsilon_{0} \rho_{p} x^{3}(R-r)^{2}} \\
& F V=0 \\
& F C=0 \\
& F F=\operatorname{CoF}\left(F S_{r}+F E+F C\right)
\end{aligned}
$$

Where $U_{\theta}, U_{r}$ is the flow velocity in the relevant direction, $\tau=\rho_{p} x^{2} / 18 \mu$ is the particle relaxation time, $\mu$ is the flow dynamic viscosity, $\varepsilon_{0}$ is the dielectric constant of free space, $R$ is the radius of cyclone cross section, CoF is the coefficient of friction, and $\rho_{p}, x, q$ is the particle density, diamter and electrosatic charge, respecitvely. (A detailed derivation of Equation (4) can be found in Appendix)

\subsubsection{Mesh-free simulation treatment}

In order to have a better resolution of the boundary layer flow velocity, a mesh-free simulation treatment was developed. First of all, the tangential velocity profile of the swirling flow in the cyclone was generated. It was set to follow the theoretical 'Rankine vortex' that contained a 'solid-body rotation' and a 'loss free vortex'.

Secondly, a boundary layer velocity profile with a constant velocity gradient was 
created to modify the tangential velocity profile in the near wall region. Although the created boundary layer only revealed the linear viscous sublayer, it was acceptable to use this created boundary layer to represent the whole boundary layer, because the purpose of creating the boundary layer velocity profile was to decrease the flow velocity rapidly in the near wall region.

The tangential velocity profile was given based on our previous CFD simulation results (Zhou et al., 2017). As shown in Figure 6, the maximum tangential velocity was $2.4 U_{i n}\left(U_{i n}\right.$ is the inlet velocity of the cyclone), the radius of the 'solid-body rotation' was $0.3 R$, and the thickness of the boundary layer was given as $0.05 R$. In addition, the radial flow velocity and the turbulence were assumed to be zero.

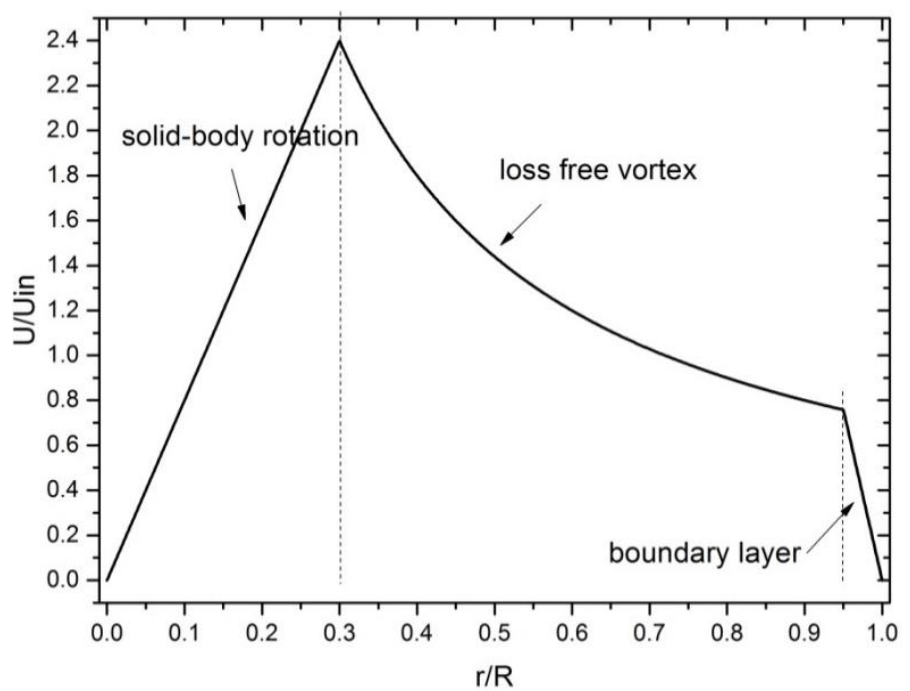

Figure 6 Generated tangential velocity profile for the mesh-free treatment

\subsubsection{Simulation conditions}

A case-study approach was adopted to determine factors that may affect the particle adhesion in the cyclone. Parameters used in different cases were determined based on our experimental conditions. Firstly, some basic parameters were given. The particle density and size was $2300 \mathrm{~kg} / \mathrm{m}^{3}$ and $1.13 \mu \mathrm{m}$, respectively. The flow dynamic viscosity was $1.8^{*} 10^{-5} \mathrm{~Pa}^{*} \mathrm{~s}$. The elementary charge was $1.60217662^{*} 10^{-19} \mathrm{C}$. The dielectric constant of free space was $8.859 * 10^{-12} \mathrm{~F} / \mathrm{m}$. Secondly, rest parameters were set as adjustable factors, covering possible values in the experiment. They had 
different values in different cases, as listed below:

Table 3 Case-study of factors affecting the particle adhesion in cyclone

\begin{tabular}{lllllll}
\hline & $r_{0}(\mathrm{~mm})$ & $R(\mathrm{~mm})$ & $q(\mathrm{C})$ & $U_{\text {in }}(\mathrm{m} / \mathrm{s})$ & CoR & CoF \\
\hline Case 0 & $0.1 R$ & 10 & $100 e$ & 30 & 0.5 & 0.5 \\
Case 1 & $0-R$ & 10 & $100 e$ & 30 & 0.5 & 0.5 \\
Case 2 & $0.5 R$ & $3.3-17.5$ & $100 e$ & 30 & 0.5 & 0.5 \\
Case 3 & $0.5 R$ & 10 & $0-200 e$ & 30 & 0.5 & 0.5 \\
Case 4 & $0.5 R$ & 10 & $100 e$ & $10-50$ & 0.5 & 0.5 \\
Case 5 & $0.5 R$ & 10 & $100 e$ & 30 & $0-1$ & 0.5 \\
Case 6 & $0.5 R$ & 10 & $100 e$ & 30 & 0.5 & $0-1$ \\
\hline
\end{tabular}

${ }^{*} r_{0}$ is the initial radial location of the particle, $e$ is the elementary charge

'Case 0' was designed to study the particle trajectory in the cyclone, aiming to understand the typical particle dynamic motion. 'Case 1' to 'Case 6' were designed to investigate the effect of the particle initial location, the effect of the radius of the cyclone, the effect of the particle charge, the effect of the inlet velocity of the cyclone, the effect of the CoR and the effect of the CoF, respectively.

The time step during different stages of the particle dynamic motion was adjusted to meet the requirement of different particle velocity in different stages. Typically, the time step was $1 / 10$ of the particle relaxation time. The stopping criterion for the simulation was the time that the particle could move after the first collision occurring. It was set to be $10^{4}$ times the particle relaxation time. In this work, the simulation was conducted by software Matlab 2012a.

\section{Results and discussion}

\subsection{Simulation results}

\subsubsection{Particle dynamic motion in the cyclone}

The simulation of 'Case 0' was carried out to have an idea of the particle dynamic motion in the cyclone. As shown in Figure 7(b), the particle entered the boundary 
layer after being released for a short time (around $0.005 \mathrm{~s})$. Within this period (< $0.005 \mathrm{~s})$, the tangential location and the radial location of the particle had a large amount of increment, as shown in Figure 7(a) and Figure 7(b). Such a large increment was due to the relative high particle velocity in the 'Rankine vortex', as shown in Figure 7(c) and Figure 7(d).

After entering the boundary layer $(>0.005 \mathrm{~s})$, a considerable amount of growth in the particle tangential location and a fall of the particle tangential velocity was found, but the particle radial velocity was close to zero and the radial location almost remained the same.

Interestingly, after the first hit occurring, the particle almost had no movement in the scale of the radius of the cyclone. This finding suggested that the majority of the particle transportation in the cyclone happened when the particle was not on the wall surface. The spiral particle trajectory in the cyclone is shown in Figure 8. After being released near the centre of the cyclone, the particle moved spirally towards the wall.

In addition, forces on the particle, written in the form of acceleration, were monitored during the simulation, as shown in Figure 9(a) and Figure 9(b). It can be seen that the tangential aerodynamic force $\left(F S_{\theta}\right)$ started at a high level and then decreased, as the particle velocity increased from zero to the flow velocity. When the particle velocity had exceeded the flow velocity, the tangential aerodynamic force turned to be negative. After the first hit occurring, it switched from the negative to positive, because the particle velocity was smaller than the flow velocity. As the particle-wall collision occurred repeatedly, the tangential aerodynamic force would fluctuate after the collision.

Meanwhile, the radial aerodynamic force $\left(F S_{r}\right)$ and the centrifugal force $(F C)$ had almost the same trend but the opposite value before the first hit occurring. It was because the radial flow velocity was assumed to be zero, so that the centrifugal force was balanced by the radial aerodynamic force. However, after the first hit, the balance was broken down that the centrifugal force was smaller than the radial aerodynamic 
force, because the particle velocity was relatively small. In addition, it was found that the electrostatic force $(F E)$ increased from zero to a considerable level rapidly, when the particle was approaching the wall. The reason was that the electrostatic force was in inverse proportion to the particle radial distance to the wall. As the centrifugal force and radial aerodynamic force were far smaller than the electrostatic force after the first hit, the friction $(F F)$ was mainly derived from the electrostatic force.

a

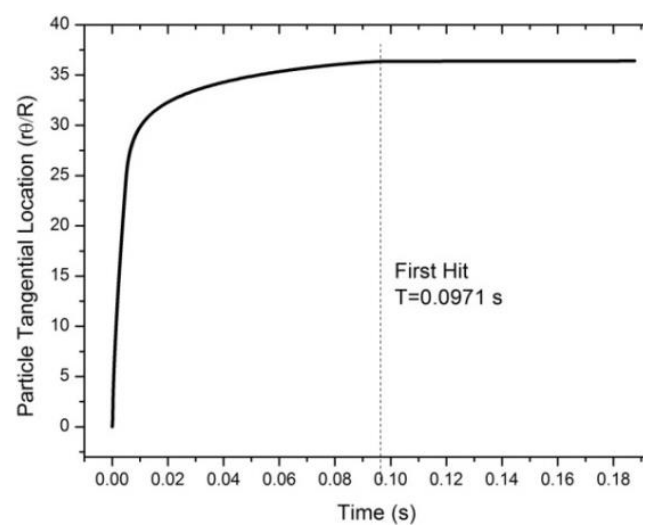

c

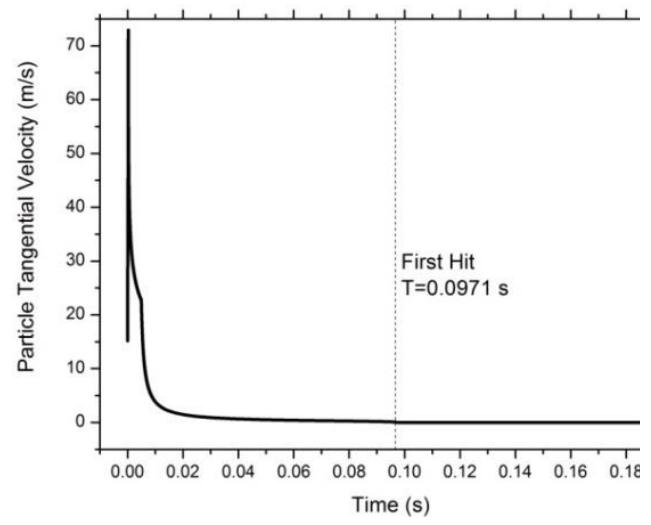

b

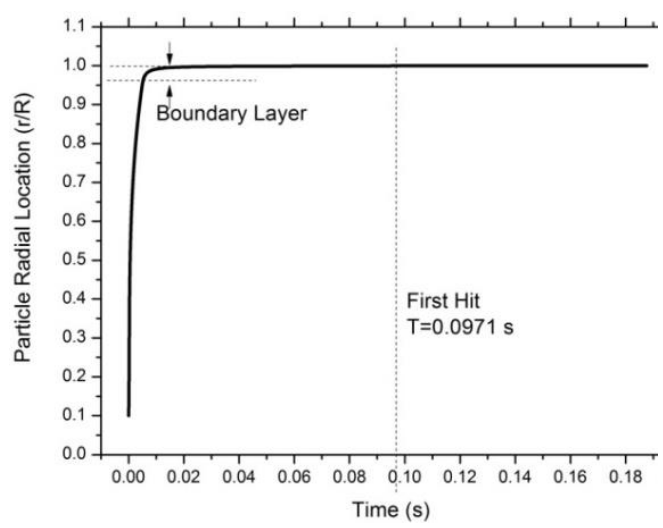

d

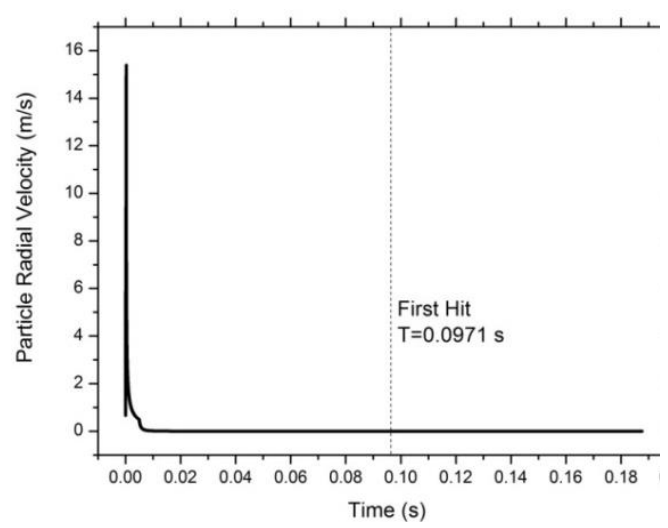

Figure 7 2D mesh-free simulation results of 'Case 0' on particle location and velocity (a) tangential location, (b) radial location, (c) tangential velocity, (d) radial velocity 


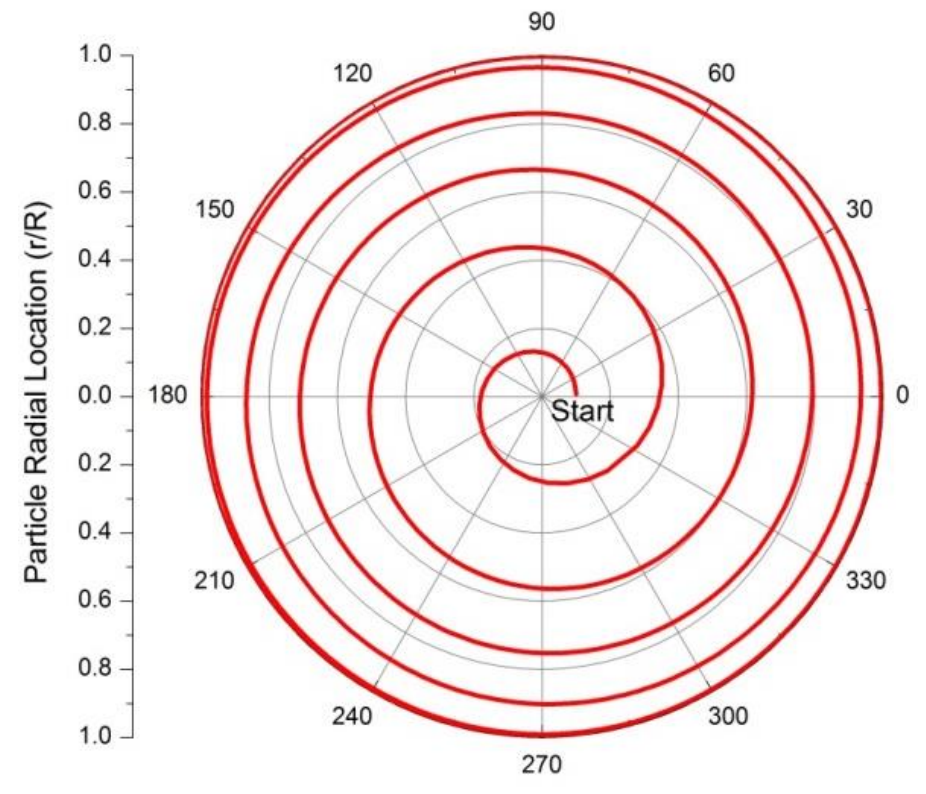

Figure 82D mesh-free simulation results of 'Case 0 ' on particle trajectory

a

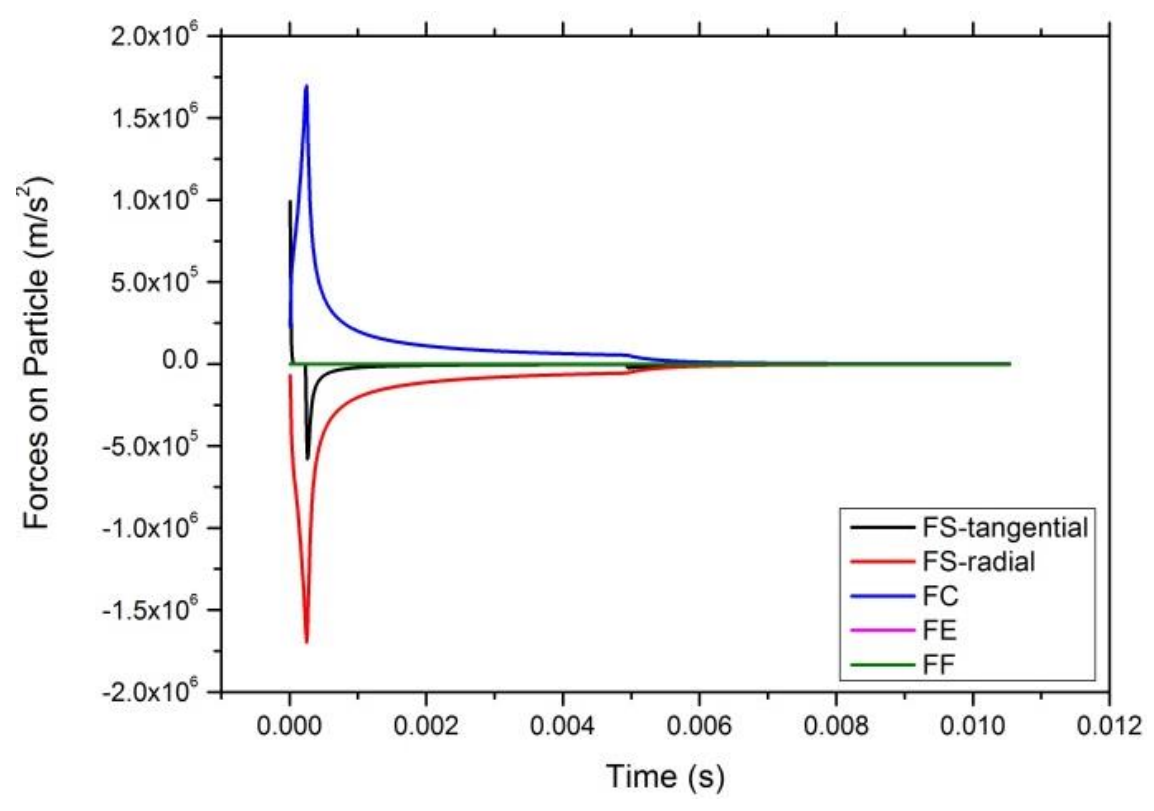




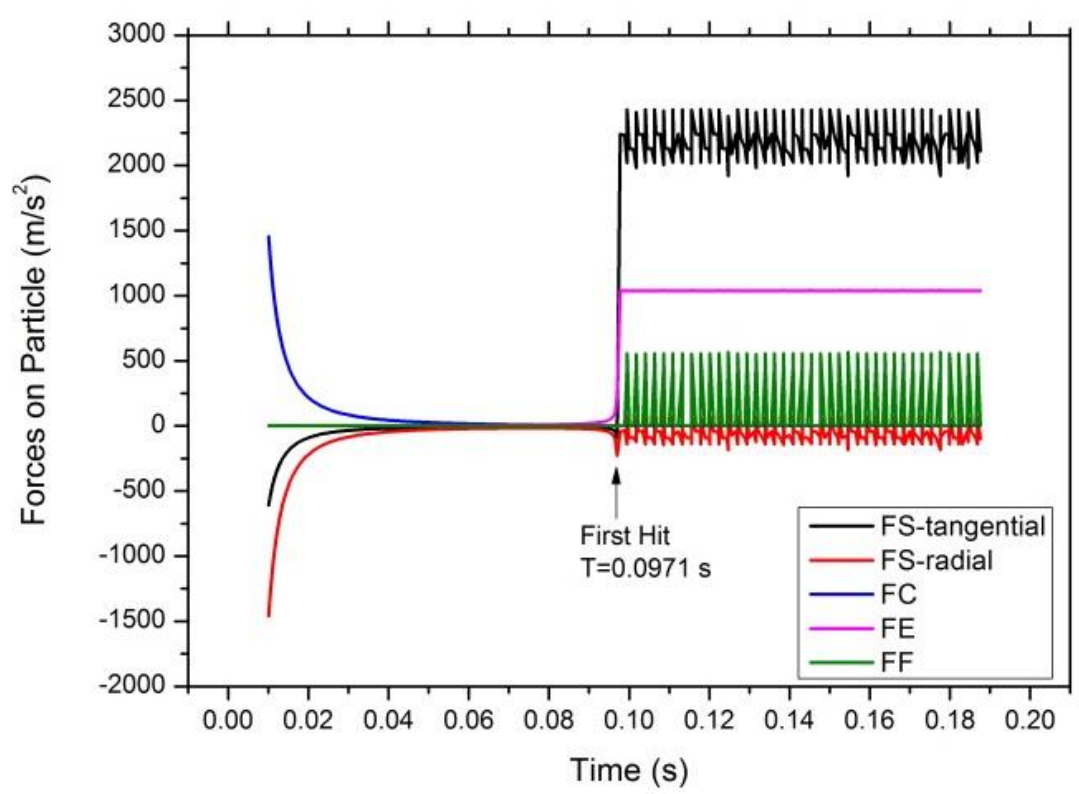

Figure 9 2D mesh-free simulation results of 'Case 0' on forces on particle (a) before first hit, (b) after first hit

\subsubsection{Factors affecting the particle adhesion}

An indirect indicator describing the ability of the particle detachment from the wall surface was chosen to quantify the particle adhesion. This indirect indicator was defined as the tangential distance that the particle could move after the first hit. It was normalized to the particle size, which could be written by $\delta(r \theta) / x$. In the same time period after the first hit, if the particle had a shorter tangential distance, it meant that the particle was closer to the state of the adhesion. Otherwise, the particle was unlikely to form the adhesion, if the particle had a longer tangential distance.

\subsubsection{Effect of particle initial location}

The simulation of 'Case 1' was conducted to examine the effect of the particle initial location. Results of the effect of the particle initial location are shown in Figure 10(a). It is clearly seen that the initial location does not affect the particle tangential distance after the first hit. The reason was that the particle velocity before the first hit did not change with the initial location, as shown in Figure 10(b). It was because of the boundary layer that damped the particle velocity, no matter what the initial 
location was.
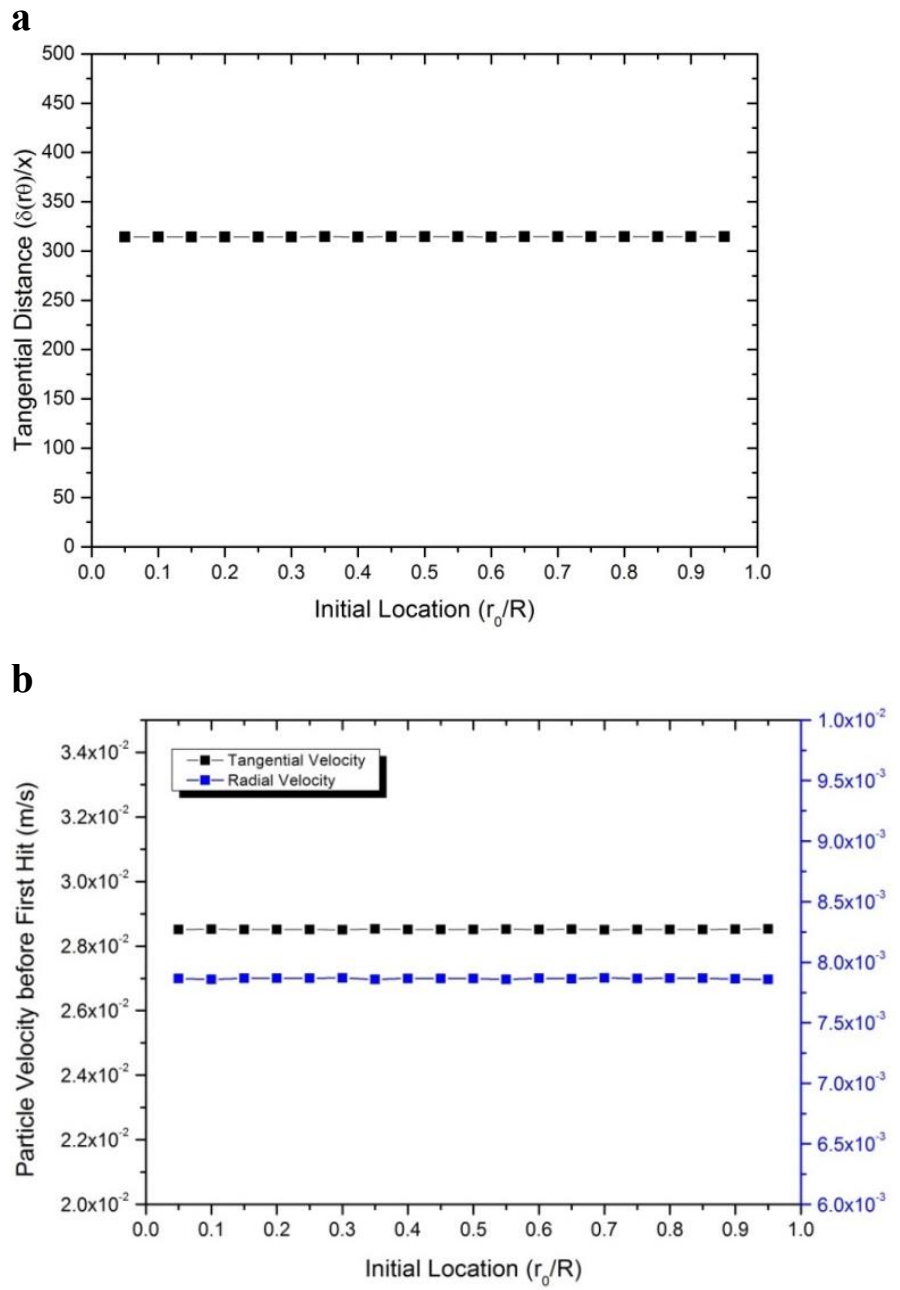

Figure 10 Effect of initial location ('Case 1') (a) tangential distance (b) particle velocity before first hit

\subsubsection{Effect of radius of the cyclone separator}

The simulation of 'Case 2' was conducted to evaluate the effect of the radius of the cyclone. Results of the particle tangential distance after the first hit and the particle velocity before the first hit are demonstrated in Figure 11(a) and (b), respectively. It can be seen that the tangential distance decreased with the increasing in the radius of the cyclone. The reason was that the velocity gradient in the boundary layer decreased as the thickness of the boundary layer increased with the increasing radius of the cyclone. This finding suggested that a smaller sized cyclone would have less particle 
adhesion, due to the higher velocity gradient in the boundary layer. The velocity gradient in the boundary layer also affected the particle tangential velocity before the first hit. However, the particle radial velocity remained the same, as the radial flow velocity was constant.

$\mathbf{a}$

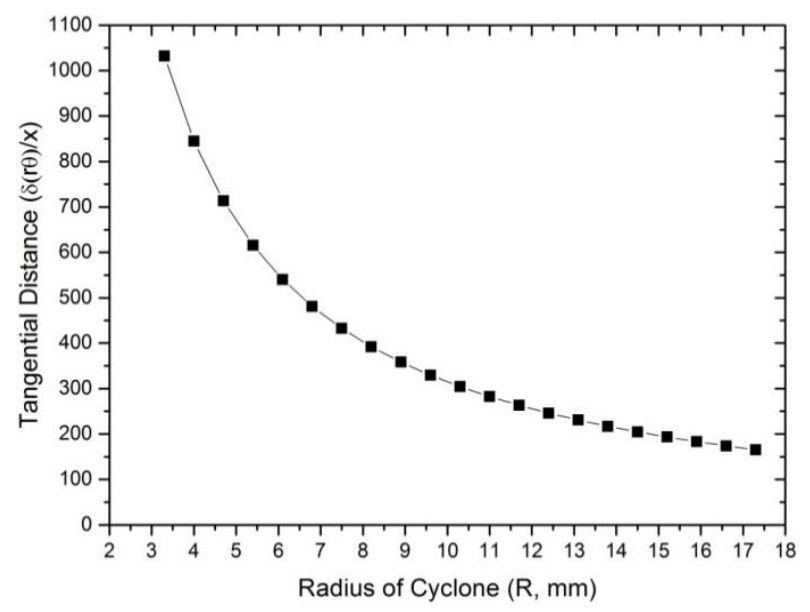

b

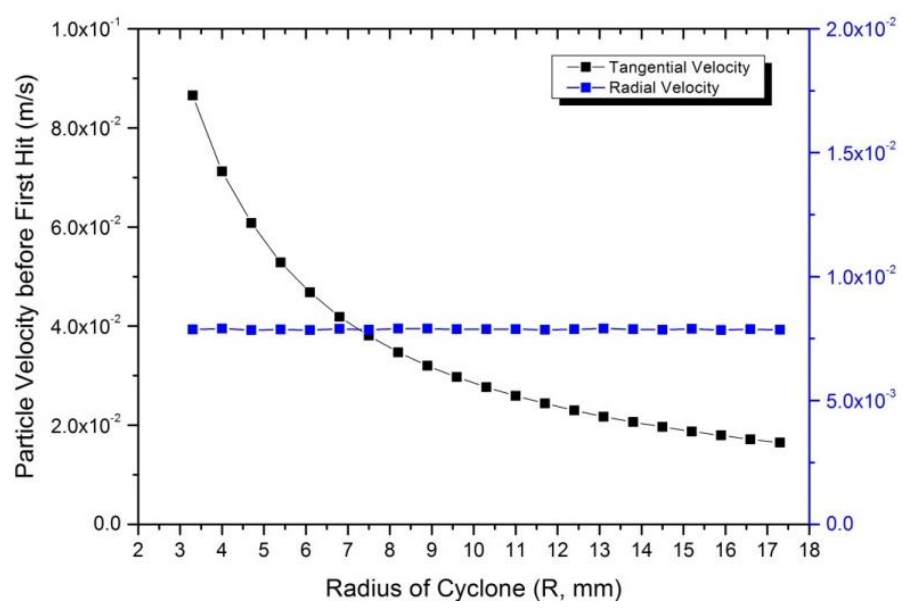

Figure 11 Effect of radius ('Case 2') (a) tangential distance, (b) particle velocity before first hit

\subsubsection{Effect of particle charge}

The simulation of 'Case 3' was conducted to help understand the effect of the particle charge. Results of the particle tangential distance are shown in Figure 12(a). It can be seen that the tangential distance decreased as the absolute value of the particle charge 
increased. It was obvious that the sign of the particle charge did not affect the results, because the electrostatic force was proportional to the square of the particle charge.

What stands out in this study was the rapid growth of the tangential distance when the particle charge was close to zero. The reason was that, when the particle charge was zero, there was almost no friction between the particle and the wall surface. Thus, the particle acted like hovering on the wall and could move far in a tangential direction.

Meanwhile, the particle velocity increased with the increasing particle charge, as shown in Figure 12 (b). It was because the electrostatic charge force pushed the particle move towards the wall, resulting in a high particle radial velocity. Furthermore, as the particle had a high radial velocity, the particle tangential velocity did not have enough time to be damped to the local low flow velocity, resulting in a high particle tangential velocity. It was noted that the friction increased with the increasing particle charge as well. As a whole, there was a decline of the particle tangential distance when the particle charge increased. 


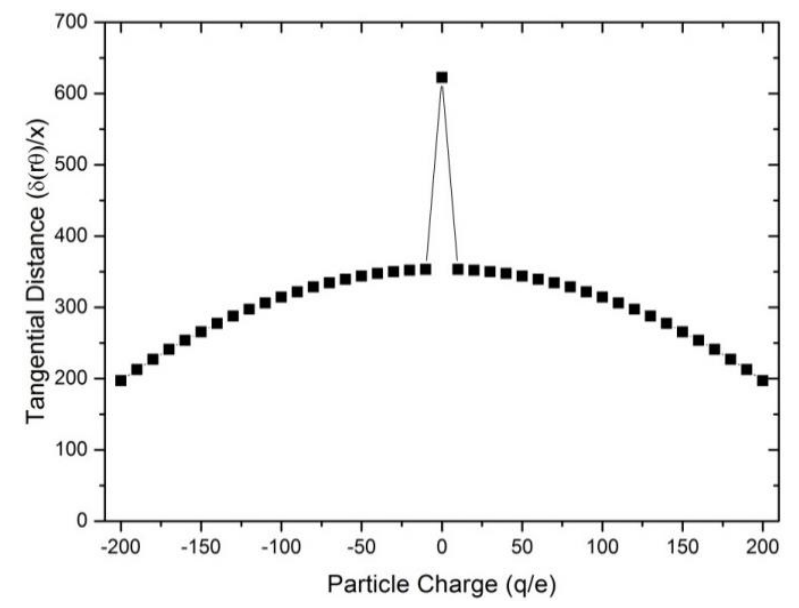

b

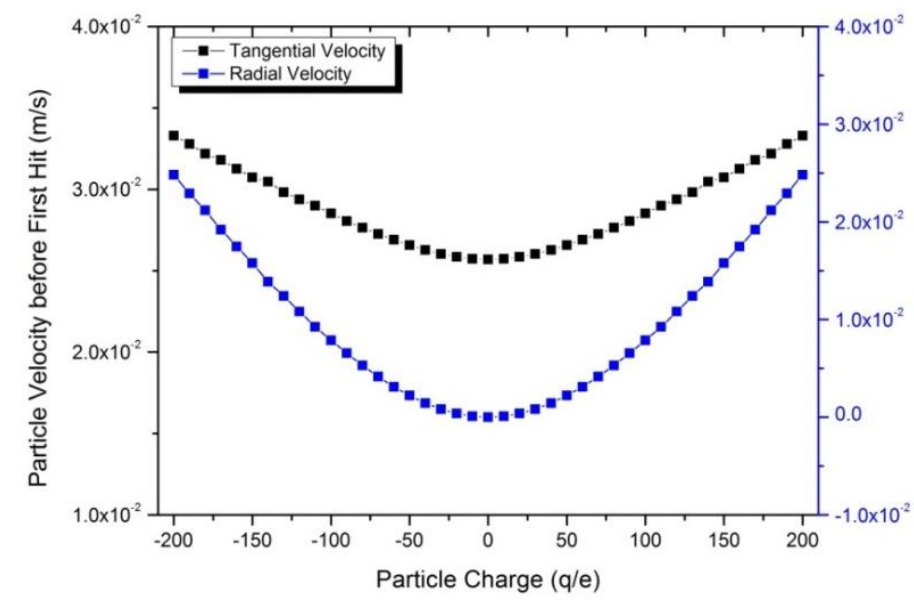

Figure 12 Effect of particle charge ('Case 3') (a) tangential distance, (b) particle velocity before first hit

\subsubsection{Effect of inlet velocity}

The simulation of 'Case 4' was conducted to assess the effect of the inlet velocity. As shown in Figure 13(a), the particle tangential distance increased linearly with the increase of the inlet velocity. As the tangential distance is proportional to particle velocity, which is almost equal to air flow velocity in the boundary layer, the tangential distance is proportional to air flow velocity in the boundary layer. Since we model the air flow velocity profile using the reference value of inlet velocity, the tangential distance increased linearly with the inlet velocity. Meanwhile, the particle 
tangential velocity before the first hit also had a linear growth with the increasing of the inlet velocity and the radial velocity was constant, as shown in Figure 13(b).

a

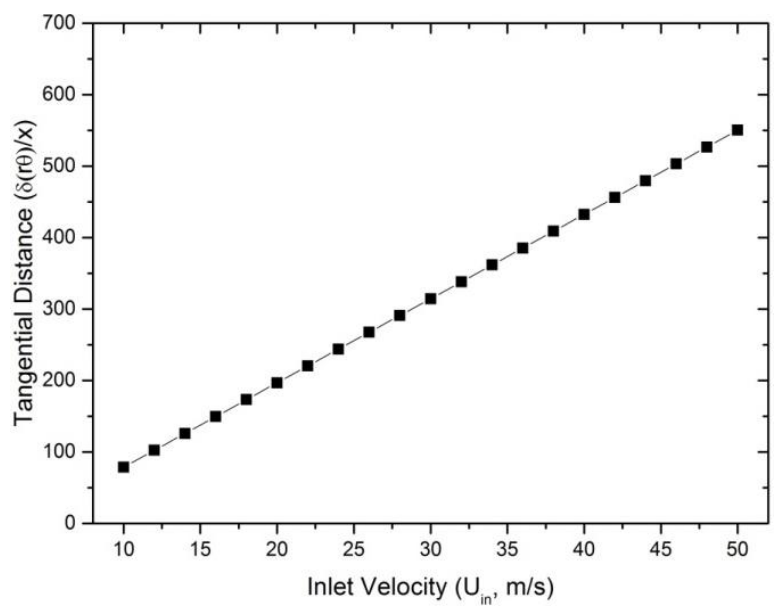

b

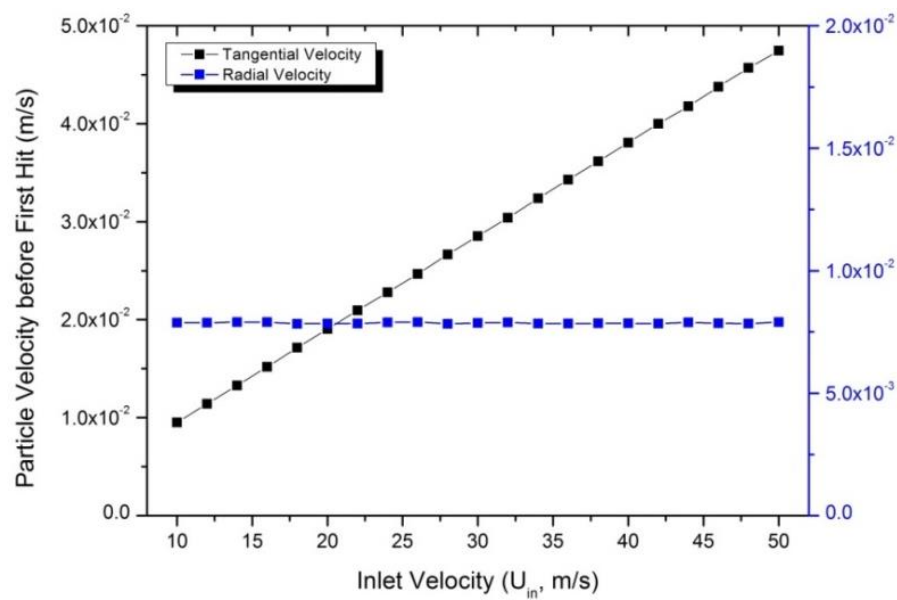

Figure 13 Effect of inlet velocity ('Case 4') (a) tangential distance, (b) particle velocity before first hit

\subsubsection{Effect of coefficient of restitution}

The simulation of 'Case 5' was conducted to obtain the information on the effect of the coefficient of restitution (CoR). Results of the particle tangential distance and the particle velocity are shown in Figure 14(a) and Figure 14(b), respectively. It can be 
seen that the tangential distance increased as the $\mathrm{CoR}$ increased. The incline ratio of the tangential distance also increased with the increasing of the CoR. The reason was that the CoR affected the collision between the particle and wall. With a larger CoR the particle can recover with a higher velocity after the hit, resulting in a higher radial distance and a longer tangential distance that the particle can move. Meanwhile, the particle velocity before the first hit did not change with the CoR, because the CoR only affected the particle motion after the hit.

$\mathbf{a}$

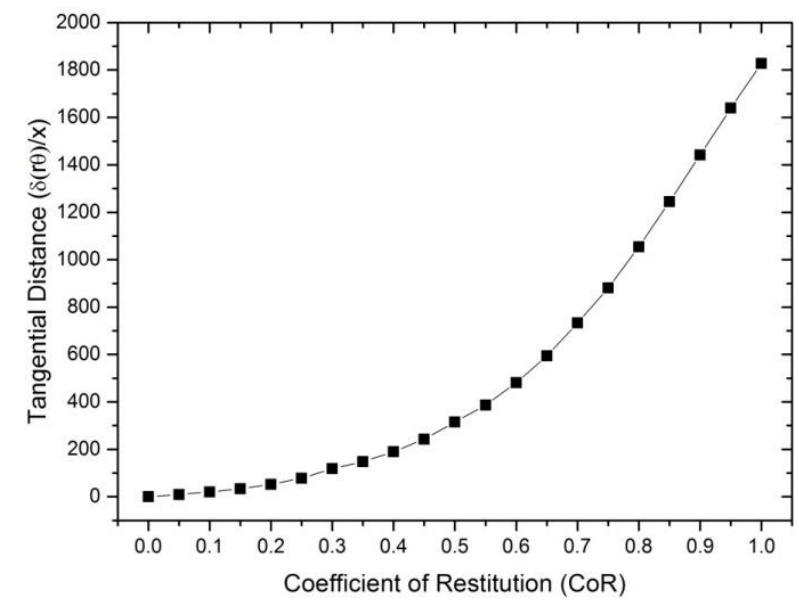

b

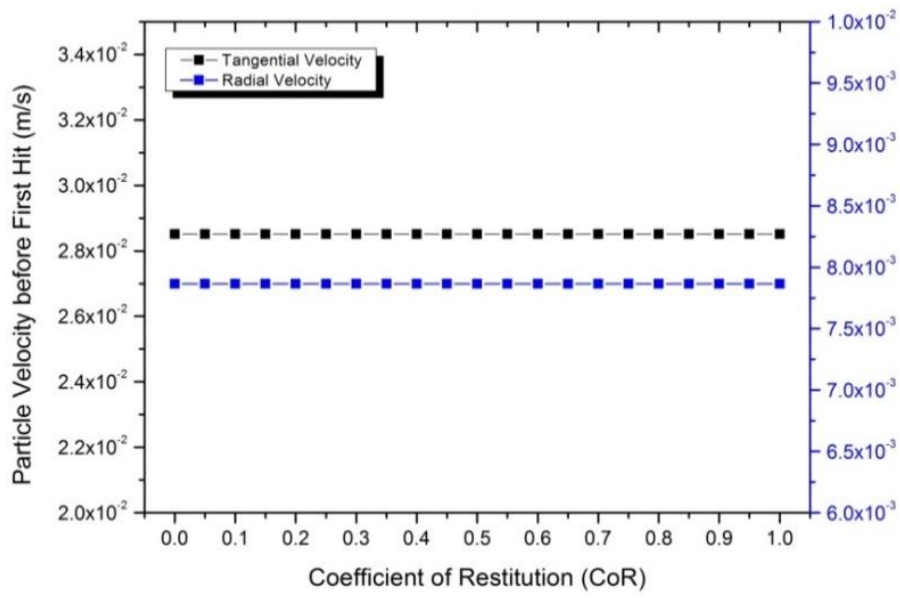

Figure 14 Effect of CoR ('Case 5') (a) tangential distance, (b) particle velocity before first hit

\subsubsection{Effect of coefficient of friction}

The simulation of 'Case 6' was conducted to provide the illustrations of the effect of 
the coefficient of friction $(\mathrm{CoF})$. As shown in Figure 15(a), the tangential distance decreased linearly as the CoF increased. It was because the friction was proportional to the CoF. Meanwhile, the particle velocity before the first hit did not change with the $\mathrm{CoF}$, as the CoF only affected the particle motion after the hit, see Figure 15(b).

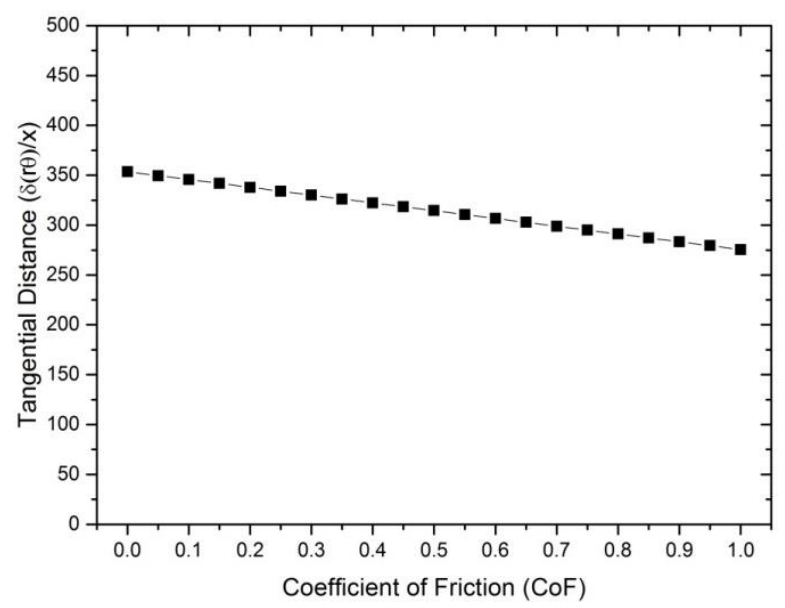

b

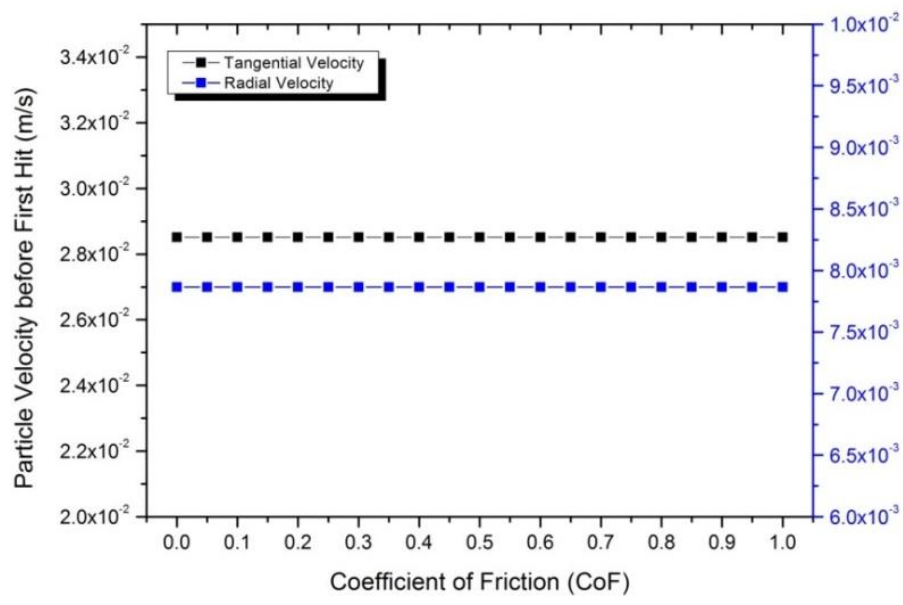

Figure 15 Effect of CoF ('Case 6') (a) tangential distance (b) particle velocity before first hit

\subsection{Validation of simulation}

\subsubsection{Effect of particle charge}


According to the simulation results, there was a relative large difference of the particle adhesion between the particle with charge and the particle without charge. In order to validate simulation results, the effect of particle charge $(q)$ was studied at the charged condition, compared with the uncharged condition.

To isolate the effect of the particle charge, the flow rate of the cyclone was fixed at $105 \mathrm{~L} / \mathrm{min}$ in the experiment, resulting in an inlet velocity of $30 \mathrm{~m} / \mathrm{s}$. Four levels of particle load rates were tested in the experiment. They were the 'low level', 'medium level', 'high level' and the 'very high level', corresponding to $0.029 \mathrm{~g} / \mathrm{min}, 0.063$ $\mathrm{g} / \mathrm{min}, 0.078 \mathrm{~g} / \mathrm{min}$, and $0.101 \mathrm{~g} / \mathrm{min}$, respectively.

The cumulative particle charge was measured by the digital coulomb meter. The results are shown in Figure 16. It can be seen that the cumulative particle charge was close to zero at the uncharged condition. However, at the charged condition, the cumulative particle charge increased almost linearly as the time progressed until it reached the maximum measurement range of the digital coulomb meter.

The measured particle charge could be estimated by following equations:

$$
\begin{aligned}
& Q_{c}=\sum_{t=0 \min }^{t=20 \min } \dot{N} q \\
& \dot{m}=\frac{\rho_{p} \pi x^{3}}{6} \dot{N}
\end{aligned}
$$

Where $Q_{c}$ is the cumulative particle charge, $\dot{N}$ is the number flux rate of particles, and $\dot{m}$ is the mass flux rate of particles.

Based on experimental results, the average single particle charge $(q)$ was about $38 e$ and $1 e$ for the charged and uncharged condition, respectively. It was noted that $1 e$ was regarded as zero charge because it was not able to achieve absolute zero charge in the experiment.

As the inlet velocity of the cyclone was controlled to be $30 \mathrm{~m} / \mathrm{s}$, the average pressure 
drop of the cyclone in 20 minutes' test was similar for both conditions, shown in Figure 17. It was found the average pressure drop was unlikely to be affected by the particle load rate, which was not the same as Chu et al. (2011) predicted in CFD. This was probably due to particle size and property were different in our experiment. In cyclone, particle-air interaction resulted in the change of pressure drop. Since particleair interaction was coupled with particle-particle interaction, it was likely that changes in particle size and property would lead to different pressure drops.

After the experiment, the amount of the particle adhesion was weighed for both conditions, as shown in Figure 18. The weight of the particle adhesion at the uncharged condition was close to zero regardless of particle load rate, which meant that there was no particle adhesion occurring. At the charged condition, the weight of the particle adhesion increased with the particle load rate. Compared with the simulation results (see Figure 12(a)), results of the experiment confirmed that the particle charge was one of factors that affected the particle adhesion and a higher particle charge resulted in more particle adhesion.

It was noted that Houben (2011) found that there was a critical particle load rate (equal to $0.252 \mathrm{~g} / \mathrm{min}$ in our experiment) for the particle adhesion. Within the critical particle load rate, particle adhesion increased with particle load rate. After exceeding the critical particle load rate, particle adhesion decreased. Since our highest particle load rate was $0.101 \mathrm{~g} / \mathrm{min}$ and our experimental results showed that particle adhesion increased with particle load rate, on this point, the experimental results agreed well with Houben (2011) that particle adhesion increased with particle load rate within the critical particle load rate $(0.252 \mathrm{~g} / \mathrm{min})$. 


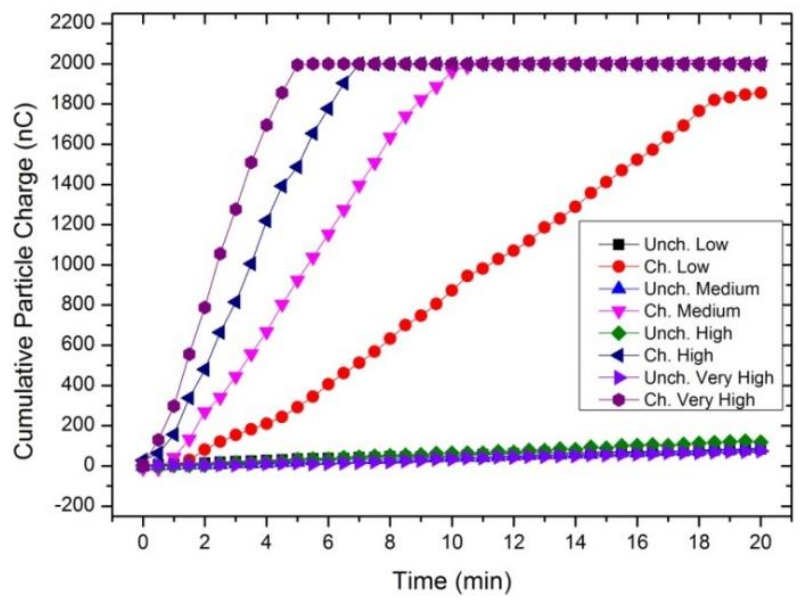

Figure 16 Measured particle charge at different particle load rate (flow rate 105 $\mathrm{L} / \mathrm{min})$

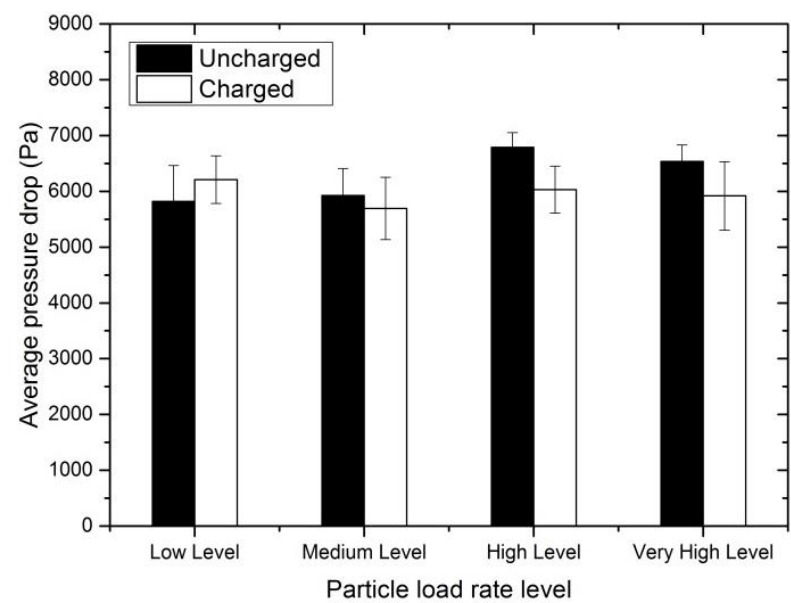

Figure 17 Pressure drop of cyclone for charged condition and uncharged condition at different particle load rate (flow rate $105 \mathrm{~L} / \mathrm{min}$ )

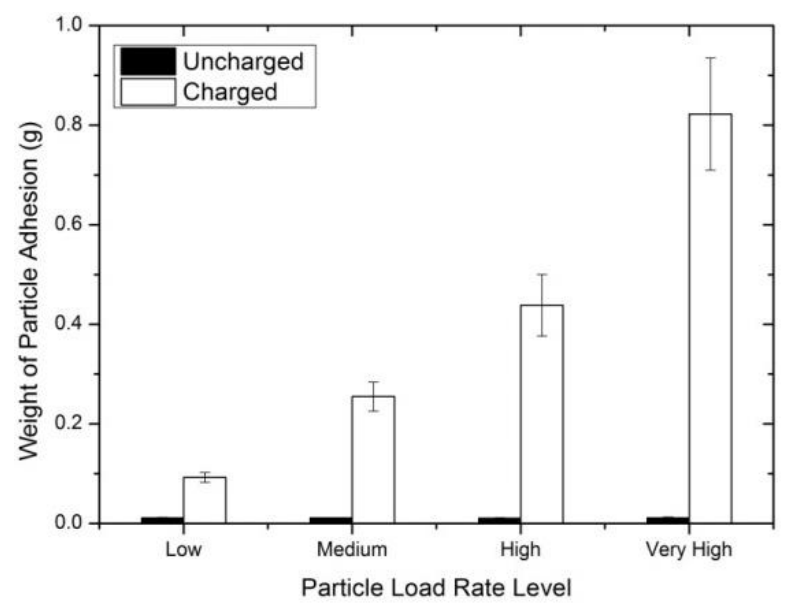

Figure 18 Weight of particle adhesion for charged condition and uncharged condition 
at different particle load rate (flow rate $105 \mathrm{~L} / \mathrm{min}$ )

\subsubsection{Effect of inlet velocity}

In order to examine the effect of inlet velocity $\left(U_{i n}\right)$, particle charge and particle load rate was fixed at charged condition and 'very high' level of particle load rate, i.e. particle charge was $38 e$ and particle load rate was $0.101 \mathrm{~g} / \mathrm{min}$. The flow rates were $105 \mathrm{~L} / \mathrm{min}, 115 \mathrm{~L} / \mathrm{min}, 125 \mathrm{~L} / \mathrm{min}, 135 \mathrm{~L} / \mathrm{min}$ and $150 \mathrm{~L} / \mathrm{min}$, respectively, resulting in the inlet velocity of $30 \mathrm{~m} / \mathrm{s}, 33 \mathrm{~m} / \mathrm{s}, 36 \mathrm{~m} / \mathrm{s}, 39 \mathrm{~m} / \mathrm{s}$ and $43 \mathrm{~m} / \mathrm{s}$, respectively. As the inlet velocity changed, the average pressure drop increased with the inlet velocity, shown in Figure 19.

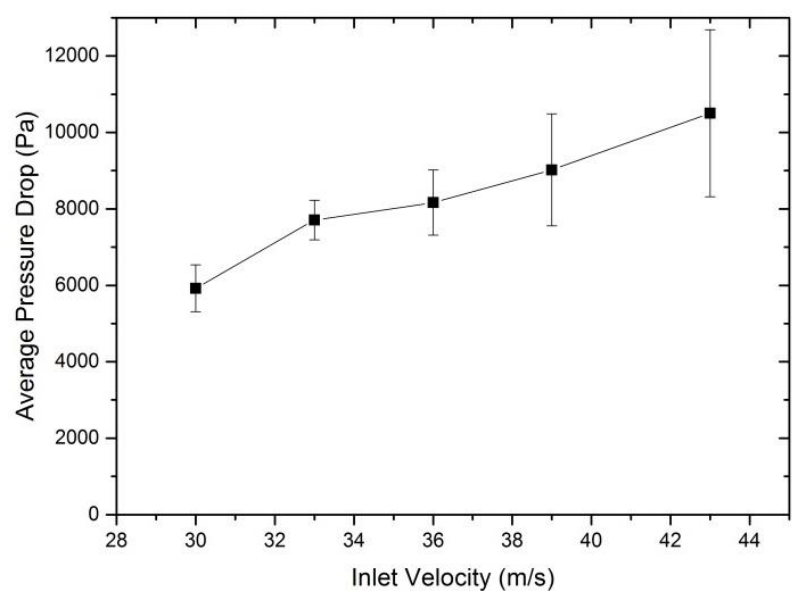

Figure 19 Pressure drop of cyclone at different flow rates (particle load rate 0.101 $\mathrm{g} / \mathrm{min})$

After the experiment, the weight of the particle adhesion was measured, as shown in Figure 20. Compared with the simulation results (see Figure 13(a)), it was confirmed that the inlet velocity affected particle adhesion and a higher inlet velocity reduced the particle adhesion, which was the same as the simulation. It was noted that Houben (2011) also found that the particle adhesion decreased as inlet velocity increased. 


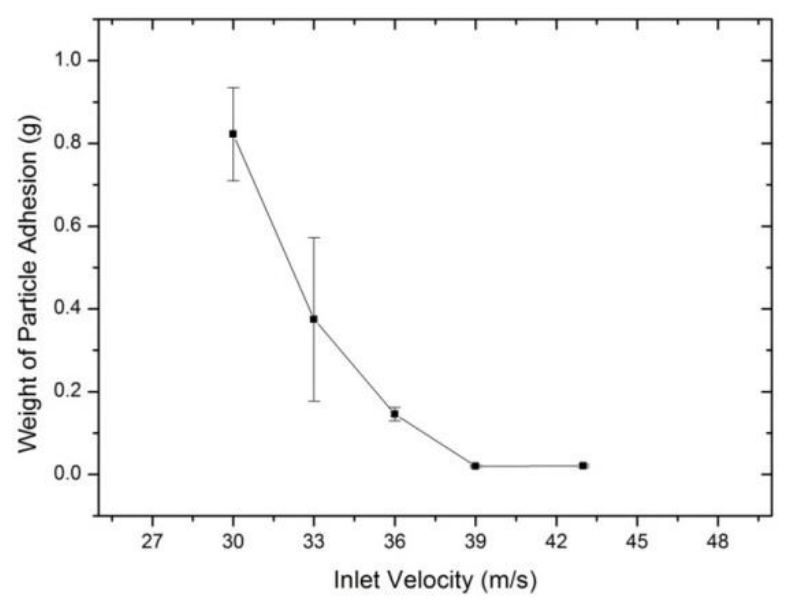

Figure 20 Weight of particle adhesion at different flow rates (particle load rate 0.101

$$
\mathrm{g} / \mathrm{min})
$$

\subsection{Discussion}

In previous study, it was believed that the centrifugal force acting on particle on the wall surface would be one of the causes of wall supporting force, especially when the particle was becoming larger (Wang et al., 2006). However, according to our simulation results, the centrifugal force was less effective when the particle was in the boundary layer, as the particle velocity in boundary layer was small.

It was also found that most of the particle transportation in the cyclone occurred during the flying stage. Only a minor amount of the particle transportation was found when the particle was in the other stages. It meant that the electrostatic force was not that important on the transportation of the particle in the cyclone. However, as the friction force was mainly derived from the electrostatic force, the electrostatic force played an important role on the particle adhesion.

Moreover, according to results of the case-study approach, factors affecting the particle adhesion in the cyclone were identified. The effect of the particle initial location was eliminated, because the particle velocity before the first hit did not change with the initial location. The radius of the cyclone, particle charge, inlet velocity, CoR and the CoF were found to affect the particle adhesion. In general, a small radius of cyclone with a high inlet velocity and high CoR can have less particle 
adhesion, by essentially increasing the particle velocity in contact with the wall. In contrast, a large radius of cyclone with a high particle charge and high CoF can have more particle adhesion, by increasing the friction force in essence.

Experimental investigations on the effect of the particle charge and inlet velocity were conducted so as to validate the simulation. According to pictures of the particle adhesion and the weight of the particle adhesion, the particle adhesion was found to increase with the increasing of the particle charge. In addition, the experiment results showed that the particle adhesion decreased as the inlet velocity increased. Both the results of the particle charge test and inlet velocity test agreed well with our simulation results. Therefore, the simulation was validated by the experiment.

\section{Conclusion}

A 2D model of the single particle dynamic motion in the cyclone was proposed, including the flying stage, the collision stage and the friction stage. Based on this model, a 2D mesh-free simulation of the single particle was developed and conducted, aiming to understand how the particle adhesion occurs in the cyclone. Results of the 2D mesh-free simulation were validated by the experiment. The findings of the $2 \mathrm{D}$ mesh-free simulation suggested that:

- The boundary layer was important for the particle adhesion in the cyclone

- The centrifugal force was less effective in the particle adhesion

- The electrostatic force was critical in the particle adhesion

- Most of the particle transportation in the cyclone occurred at the flying stage

- The initial location of the particle did not affect the particle adhesion

- The radius of the cyclone, the particle charge, the inlet velocity, the CoR and the CoF were found to affect the particle adhesion

- A less charged particle in a small cyclone with a high inlet velocity would 
have less particle adhesion, when the CoR was large and the CoF was small. Otherwise, there would be more particle adhesion.

\section{Future work}

The findings of this study were limited to the simplifications in the simulation, as real conditions of the particle adhesion in the cyclone were much more complicated, including the unsteady flow, turbulence, particle-particle interaction, the non-spherical geometry of the particle and the wall surface property. We can forecast the importance of those real conditions on the particle transportation in the cyclone. For example, if the particle was lifted by the turbulence of the radial flow, the particle might be suspending above the wall, which would lead to zero particle adhesion, as the particle was not on the wall surface.

It is difficult to fully understand particle behaviour in cyclone. However, future work can be carried on refining the simulation with more accurate boundary layer velocity profile, which includes the viscous sublayer, log law layer and outer layer. Models to predict the van der Waals force and capillary force can be added to the simulation by using proper physical parameters of particle and wall surface. Furthermore, the simulation code can be written into CFD software, which will consider the flow velocity in other directions and turbulence as well.

\section{Acknowledgement}

The author would like to acknowledge the Dyson Ltd. for funding this research. Also thanks for James Allan in National Centre for Atmospheric Science (NCAS) for the assistance with particle size measurements.

\section{References}

[1] O’Callaghan, D., Cunningham, P. (2005) "Modern process control techniques in the production of dried milk products - a review", Lait 85: 335-342

[2] Huang, M., Zhou, P. and Yang, J. (2013) Anti-blockage device of cyclone 
separator. Patent CN203454113U.

[3] He, S., Zeng, J. and Li, S. (2014) High-temperature cyclone separator antiblocking apparatus and method. Patent CN104147858A

[4] Mozley, R. H. (1979). Cyclone separator. Patent US4148723

[5] Yuu, S., Jotaki, T., Tomita, Y., \& Yoshida, K. (1978). The reduction of pressure drop due to dust loading in a conventional cyclone. Chemical Engineering Science, 33(12), 1573-1580.

[6] Bogodage, S. G., and Leung, A. Y. T. (2016). Improvements of the cyclone separator performance by down-comer tubes. Journal of Hazardous Materials,311, $100-114$.

[7] Houben, J. J. H. (2011). Experimental investigations and CFD simulations on particle depositions in gas cyclone separators. Ph. D. thesis, Montanuniversitaet Leoben.

[8] Jones, R., Pollock, H. M., Cleaver, J. A., and Hodges, C. S. (2002). Adhesion forces between glass and silicon surfaces in air studied by AFM: Effects of relative humidity, particle size, roughness, and surface treatment. Langmuir, 18(21), 80458055.

[9] Bowling, R. A. (1985). An analysis of particle adhesion on semiconductor surfaces. Journal of the Electrochemical Society, 132(9), 2208-2214.

[10] Corn, M. (1961). The adhesion of solid particles to solid surfaces, I. A review. Journal of the Air Pollution Control Association, 11(11), 523-528.

[11] Cleaver, J. W., and Yates, B. (1973). Mechanism of detachment of colloidal particles from a flat substrate in a turbulent flow. Journal of Colloid and Interface Science, 44(3), 464-474.

[12] Mittal, K. L., and Jaiswal, R. (2015). Particle adhesion and removal. John Wiley 
\& Sons.

[13] Peng, W., Hoffmann, A. C., Dries, H., Regelink, M., \& Foo, K. K. (2007). Reverse flow centrifugal separators in parallel: Performance and flow pattern. AIChE journal, 53(3), 589-597.

[14] Zhou, Y., Zhong, S., \& Li, L. (2017). Characteristics and mechanisms of particle adhesion patterns in an aerodynamic cyclone. Aerosol Science and Technology (justaccepted)

[15] Song, J., Wei, Y., Sun, G. and Chen, J. (2017). Experimental and CFD study of particle deposition on the outer surface of vortex finder of a cyclone separator. Chemical Engineering Journal, 309, pp.249-262.

[16] Lim, E.W.C., Yao, J. and Zhao, Y. (2012). Electrostatic effects in pneumatic transport of granular materials. In International Journal of Modern Physics: Conference Series (Vol. 19, pp. 351-361). World Scientific Publishing Company.

[17] Wang, B., Xu, D.L., Chu, K.W. and Yu, A.B. (2006). Numerical study of gassolid flow in a cyclone separator. Applied Mathematical Modelling, 30(11), pp.13261342.

[18] Chu, K. W., Wang, B., Xu, D. L., Chen, Y. X., and Yu, A. B. (2011). CFD-DEM simulation of the gas-solid flow in a cyclone separator. Chemical Engineering Science, 66(5), 834-847.

[19] Houben, J. J. H., Weiss, C., and Raupenstrauch, H. (2012). Trajectory prediction of a point mass particle in a predefined wall-bounded flow field with the objective of estimating its wall-impact velocity. Computers \& Fluids, 57, 172-182.

[20] Hoffmann, A.C. and Stein, L.E. (2002). Gas cyclones and swirl tubes. SpringerVerlag Berlin Heidelberg.

[21] Wang, H. C. (1990). Effects of inceptive motion on particle detachment from 
surfaces. Aerosol Science and Technology, 13(3), 386-393.

[22] O'Neill, M.E. (1968). A sphere in contact with a plane wall in a slow linear shear flow. Chemical Engineering Science, 23(11), pp.1293-1298. 